\title{
Pediatric Research: Tradition and Transition
}

$\mathrm{M}$ ore than 40 years after the first issue of Pediatric Research was published, we are happy to report that the Journal is in great shape. Pediatric Research continues to publish exciting findings from laboratories and clinical research groups from around the globe. Motivated by the recently launched division into basic, translational, and clinical contributions, some of us have come to call Pediatric Research the only "bench-to-bedside" journal in pediatrics.

By initiating these and other changes, the previous Chief Editors, Sherin Devaskar and Petra Hüppi, have made Pediatric Research more accessible and cutting edge than ever before. At the same time, they have preserved the tradition of the Journal as being devoted to the publication of the best possible research. We appreciate their excellent work and acknowledge their accomplishments that pushed the Journal forward.

We know that theirs is a hard act to follow, especially in light of the two important transitions that we have before us. The first transition will be the process of bringing in a new editorial board. The second will occur in 2012, when we move the Journal to a new publisher. Once the dust has settled from these transitions, we will direct our full attention to three primary objectives that we hope to address during our tenure: 1) internationalization of the editorial board, 2) expansion of the scope and impact of the Journal, and 3) encouraging contribution from young investigators.

Internationalization. Last year, we accepted responsibility for leading a team of Section Editors and Editors, who will assist us in identifying the best articles for publication in the Journal. We are delighted that many of our superb colleagues around the world accepted the invitation to join us in this endeavor. The creation of a single, international editorial board is the first step in a transition toward a truly international research journal. Over the coming years, we pledge to continue to strive toward the inclusion of more editors outside Europe and North America.

Scope and impact. For the benefit of our readers, articles in each issue will continue to be categorized as basic, translational, and clinical investigations, and we hope to maintain a healthy balance among all three areas of research. Although still being devoted to publishing the best basic science articles in pediatric and developmental research, we encourage translational and clinical investigators from all fields of pediatrics to submit their best manuscripts to Pediatric Research. For the benefit of our authors, we have added the concept of editorial "sections," roughly defined by the traditional subdisciplines of pediatrics, each with its own strong editorial team led by a world-class expert in the field. By identifying editorial sections, we hope to expand the scope of the Journal by attracting submissions from areas, such as oncology, rheumatology, epidemiology, and public health, which have been underrepresented. We will attempt to improve the Journal's impact by further adjusting the proportion of manuscripts that enter peerreview and the final acceptance rate to a healthy level that maximizes the quality of the Journal's content while minimizing the workload for reviewers, editors, and staff.

Young investigators. Finally, we would like to think of Pediatric Research as a young journal (despite its more than $40 \mathrm{y}$ in print!). In this spirit, we would like to encourage as many young researchers as possible to submit their best work to the Journal with the hope that they will get one of their first papers accepted for publication in Pediatric Research. To this end, we are already working toward obtaining support for an annual prize for the best publication by a junior investigator. The process of preparing for the change of editorial teams has been an exciting exercise, and we thank Sherin Devaskar and Petra Hüppi, Susan Tsujimoto, Managing Editor for the Journal, Fred Suchy and Bill Hay, President and Secretary/ Treasurer, respectively, of the International Pediatric Research Foundation (IPRF), and all other IPRF trustees for their continued guidance and kind support.

We look forward to the journey ahead and to the challenges we will undoubtedly face. We will always keep in mind the wonderful fellow travelers on the journey with us, and if/when the ride becomes a little bit bumpy, we hope that you will find that the goal is worth the effort. In the meantime, enjoy the read, and we wish you all the best for a happy, healthy, and successful 2011!

OLAF DAMMANN

Floating Hospital for Children at Tufts Medical Center Boston

Massachusetts 02111

PIERRE GRESSENS Inserm, U676

Hôpital Robert Debré Paris FR-75019

France 